\title{
A refinement of Alperin's Conjecture for blocks of the endomorphism algebra of the Sylow permutation module
}

\author{
LAURENCE BARKer AND İPEK Tuvay
}

\begin{abstract}
We present a refinement of Alperin's Conjecture involving the blocks of the endomorphism algebra of the permutation module formed by the cosets of a $p$-subgroup. We prove the conjecture in two special cases where every weight module has a simple socle.
\end{abstract}

Mathematics Subject Classification. Primary 20C20.

Keywords. Weight module, Cyclic defect group, Connected module.

1. Statement of the Conjecture. Shortly after proposing his weight conjecture [2], Alperin suggested, in seminars, that one approach towards tackling the conjecture would be to examine the endomorphism algebra $\operatorname{End}_{k G}(k G / S)$ of the permutation $k G$-module $k G / S$. Here, $k$ is an algebraically closed field of prime characteristic $p$ and $S$ is a Sylow $p$-subgroup of a finite group $G$. Naehrig [10] has supplied some empirical evidence to suggest that the simple socle constituents of the regular module of $\operatorname{End}_{k G}(k G / S)$ may serve as an intermediate tool to relate the simple $k G$-modules with the weight $k G$-modules.

Recall, a weight $k G$-module is defined to be an indecomposable $k G$-module $W$ such that, letting $P$ be a vertex of $W$, then the $k N_{G}(P)$-module in Green correspondence with $W$ is the inflation of a simple projective $k N_{G}(P) / P$ module. The weak form of Alperin's Weight Conjecture [2] asserts that the number of isomorphism classes of simple $k G$-modules is equal to the number of isomorphism classes of weight $k G$-modules. The block form of Alperin's Conjecture asserts that, given a block $b$ of $k G$, then the number of isomorphism classes of simple $k G b$-modules is equal to the number of isomorphism classes of weight $k G b$-modules.

This work was completed with the support of Tübitak Scientific and Technological Research Funding Program 1001 with the grant number 114F078. 
By an easy application of Frobenius Reciprocity, every simple $k G$-module occurs in both the socle and the head of $k G / S$. The rationale for the study of $\operatorname{End}_{k G}(k G / S)$ arises from the following observation of Alperin [2, Lemma 1], which tells us that, in particular, every weight $k G$-module occurs in both the socle and the head of $k G / S$.

Lemma 1.1. (Alperin) Every weight $k G$-module occurs as a direct summand of the Sylow permutation $k G$-module $k G / S$.

We deem all $k G$-modules to be finite-dimensional. A $k G$-module $L$ is said to be connected provided $\operatorname{End}_{k G}(L)$ has a unique block. It is easy to see that a direct summand $L$ of a $k G$-module $M$ is maximal among the connected direct summands of $M$ if and only if $L=e M$ for some block $e$ of $\operatorname{End}_{k G}(M)$. When these equivalent conditions hold, we call $L$ a proper component of $M$. Plainly, any $k G$-module is the direct sum of its proper components.

We say that a $k G$-module $L$ lies in a $k G$-module $M$, written $L \dashv M$, provided that $L$ is isomorphic to the image of a $k G$-endomorphism of a direct sum of finitely many copies of $M$. This is equivalent to the condition that there exists a direct sum $M^{\prime}$ of finitely many copies of $M$ such that $L$ is isomorphic to a submodule of $M^{\prime}$ and $L$ is isomorphic to a quotient module of $M^{\prime}$. We say that $M$ is accordant provided the number of isomorphism classes of simple $k G$-modules lying in $M$ is equal to the number of isomorphism classes of weight $k G$-modules lying in $M$.

Using Lemma 1.1, it is not hard to see that, for any $p$-subgroup $P$ of $G$, the weak form of Alperin's Conjecture holds for $k G$ if and only if the permutation $k G$-module $k G / P$ is accordant.

Conjecture 1.2. For any p-subgroup $P$ of $G$, every proper component of $k G / P$ is accordant.

The next three remarks are very easy and we omit the proofs.

Remark 1.3. Given a connected $k G$-module $L$ lying in a $k G$-module $M$, then $L$ lies in a unique proper component of $M$.

Remark 1.4. Let $U$ and $V$ be connected $k G$-modules lying in a $k G$-module $M$. Then $U$ and $V$ lie in the same proper component of $M$ if and only if there exist connected $k G$-modules $W_{0}, \ldots, W_{r}$ lying in $M$ such that $W_{0} \cong U$ and $W_{r} \cong V$ and for each $1 \leq i \leq r$, there exists a non-zero $k G$-map $W_{i-1} \rightarrow W_{i}$ or $W_{i-1} \leftarrow W_{i}$.

Remark 1.5. Let $L$ and $M$ be $k G$-modules such that $L \dashv M$. Let $U$ and $V$ be connected $k G$-modules lying in $L$. Then $U$ and $V$ lie in $M$. If $U$ and $V$ lie in the same proper component of $L$, then $U$ and $V$ lie in the same proper component of $M$.

In the special case where $P$ is trivial, Conjecture 1.2 is equivalent to the block form of Alperin's Conjecture. So the next result can be interpreted as saying that Conjecture 1.2 is a refinement of Alperin's Conjecture. 
Proposition 1.6. Let $P$ and $Q$ be p-subgroups of $G$ with $P \leq Q$. If every proper component of $k G / Q$ is accordant, then every proper component of $k G / P$ is accordant.

Proof. By Frobenius Reciprocity, every simple $k G$-module lies in $k G / Q$. By Lemma 1.1, every weight $k G$-module lies in $k G / S$. But $k G / S \dashv k G / Q$, so every weight $k G$-module lies in $k G / Q$. Since $k G / Q \dashv k G / P$, the required conclusion now follows from Remark 1.5.

Therefore, if Conjecture 1.2 holds when $P=S$, then it holds for all $p$ subgroups $P$ of $G$ and, in particular, the block form of Alperin's Conjecture holds for $k G$.

Let us point out a connection with Naehrig [10]. When two indecomposable direct summands $U$ and $V$ of $k G / S$ are equivalent in the sense of [10, 4.1(b)], the corresponding principal indecomposable modules of $\operatorname{End}_{k G}(k G / S)$ lie in the same block of $\operatorname{End}_{k G}(k G / S)$, hence $U$ and $V$ lie in the same connected component of $k G / S$.

In Sect. 2, we shall illustrate the conjecture with some examples. In Sect. 3, we shall deal with two special cases. We shall show that, when $G$ has a split BN-pair of characteristic $p$, the Cabanes-Sawada Theorem immediately implies that the conjecture holds for the Sylow permutation $k G$-module. We shall also show that, letting $T$ be a Sylow $p$-subgroup of the normalizer of a cyclic defect group of a block $b$ of $k G$, then the conjecture holds for the proper components of $b k G / T$.

The conjecture originates in [3]. Though not mentioned in [4], it was one of the motives for the defect theory, in [4], for blocks of endomorphism algebras.

2. Some examples. In this section, to illustrate Conjecture 1.2 , we present the structure of the Sylow permutation module in two particular cases.

First put $p=2$ and $G=A_{7}$. Using the MAGMA source code in Zimmermann's thesis [12], it can be shown that, over the field $\mathbb{F}_{2}$ of order 2 , the 2-Sylow permutation module has the depicted structure, where $n$ denotes an $n$-dimensional simple $\mathbb{F}_{2} G$-module and $n^{*}$ denotes its dual.

$$
\begin{aligned}
& (1) \oplus(14) \oplus\left(\begin{array}{cc}
14 & 20 \\
1 & 1 \\
14 & 20
\end{array}\right) \oplus 2\left(\begin{array}{c}
20 \\
1 \\
14 \\
1 \\
20
\end{array}\right) \oplus\left(\begin{array}{c}
4^{*} \\
6 \\
4
\end{array}\right) \oplus\left(\begin{array}{c}
4 \\
6 \\
4^{*}
\end{array}\right) \oplus\left(\begin{array}{ccc}
14 & & \\
4 & 6 & \\
& & \\
& 6 &
\end{array}\right) . \\
& \oplus(6) \oplus\left(\begin{array}{cc} 
\\
6
\end{array}\right)
\end{aligned}
$$

Using Zimmermann's MAGMA routines or, alternatively, using data in Benson [5, Appendix], it can be shown that all 6 of the simple $\mathbb{F}_{2} G$-modules are absolutely simple.

Again using MAGMA or [5, Appendix], it can be shown that the indecomposable summands with Loewy length 5 are projective and therefore cannot be weight modules. The non-simple indecomposable summand with socle 6 
has vertex $V_{4}$ and has a 4-dimensional non-simple Green correspondent, so this summand is not a weight module. But the simple summand 6 and the indecomposable summands with socles 4 and $4^{*}$ all have vertex $V_{4}$ and Green correspondents that are 2-dimensional, absolutely simple, and inflated from projective modules. So those three summands are weight modules. Similarly, the simple summands 1 and 14 and the indecomposable summand with socle $14+20$ are weight modules. Evidently, the proper components of the Sylow permutation module have dimensions $1,260,54$ with 1,2, 3 isomorphism classes of simple modules and 1,2,3 isomorphism classes of weight modules lying in them.

Let us give an example where the partitioning of simple modules and weight modules into blocks of $\operatorname{End}_{k G}(k G / S)$ is much finer than the partitioning into blocks of $k G$. Using MAGMA or [5, Appendix], it is not hard to show that, for $p=3$ and $G=M_{10}$, the 3-Sylow permutation module has the structure

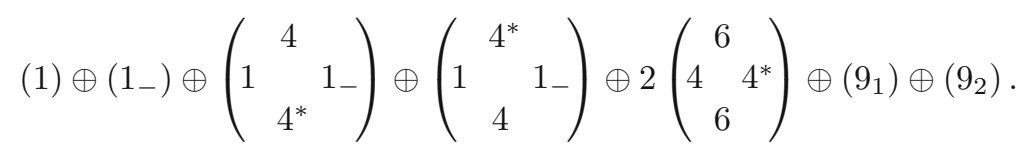

In this case, the principal block of $k G$ contains 4 of the proper components.

The authors have also confirmed that Conjecture 1.2 holds for the groups $S_{6}, A_{7}, L_{2}(25), M_{11}, J_{1}$ in characteristic 2 , for $S_{6}, S_{7}, A_{8}, L_{3}(4), L_{2}(25), M_{11}$ in characteristic 3, and for $\mathrm{McL}$ in characteristic 5. Using data in LempkenStaszewski [9], it can be shown that, in the principal 5-block of McL, three of the weight modules have socles of the form $2.250+896_{2}$ and $2.560+3038+$ $3245_{1}+3245_{2}$ and $896_{1}+3.3038$.

3. Proof in two special cases. Let us first show that the conjecture holds in the scenario of the Cabanes-Sawada Theorem.

Theorem 3.1. (Cabanes-Sawada) Suppose that $G$ has a split BN-pair of characteristic $p$. Let $S$ be a Sylow p-subgroup of $G$. Then:

1. Every indecomposable direct summand of $k G / S$ is a weight $k G$-module. Every weight $k G$-module occurs with multiplicity 1 in $k G / S$.

2. There is a bijective correspondence between the isomorphism classes of simple $k G$-modules $U$ and the isomorphism classes of weight $k G$-modules $W$ such that the isomorphism classes of $U$ and $W$ correspond provided $U \cong \operatorname{soc}(W)$.

In particular, every proper component of $k G / S$ is accordant.

Proof. This follows from Cabanes [6, Proposition 8], which says that the weak form of Alperin's Conjecture holds for $k G$, and Sawada [11, 2.8], which says that every simple $k G$-module has multiplicity 1 in $\operatorname{soc}(k G / S)$.

For another approach towards simultaneously refining Alperin's Conjecture and generalizing the Cabanes-Sawada Theorem, see [10, Section 3]. We now turn to the case of a block with a cyclic defect group. 
Theorem 3.2. Let $b$ be a block of $k G$ with a cyclic defect group D. Let $T$ be a Sylow p-subgroup of $N_{G}(D)$. Then every proper component of bkG/T is accordant.

Proof. Erdmann's Theorem [7] asserts that, given a simple $k G$-module $V$ with cyclic vertex $Q$, then $Q$ is the defect group of the block of $k G$ containing $V$. Hence, using the compatibility of the Green correspondence and the Brauer correspondence, as recorded in Alperin [1, 14.4], it is easy to show that every simple $k G b$-module and every weight $k G b$-module has vertex $D$.

We may assume that $D$ is non-trivial. Let $E$ be the smallest non-trivial subgroup of $D$. Suppose that $E \unlhd G$. Given a subgroup $L$ of $G$ containing $E$, we write $\bar{L}=L / E$. Let $\bar{b}$ be the image of $b$ under the canonical epimorphism $k G \rightarrow k \bar{G}$. The simple $k G b$-modules, all of which have vertex $D$, are the inflations of the simple $k \bar{G}$-modules, all of which have vertex $\bar{D}$. Writing $\bar{b}=$ $\sum_{i} b_{i}$ as a sum of blocks $b_{i}$ of $k \bar{G}$, then all the blocks $b_{i}$ have defect group $\bar{D}$. Since $\bar{T}$ is a Sylow $p$-subgroup of the group $N_{\bar{G}}(\bar{D})=\overline{N_{G}(D)}$, an inductive argument on $|D|$ allows us to assume that every proper component of $\bar{b} k \bar{G} / \bar{T}$ is accordant. Observing that $\bar{b} k \bar{G} / \bar{T}$ inflates to $b k G / T$, we deduce that $b k G / T$ is accordant in the case $E \unlhd G$.

Now suppose that $E$ is not normal in $G$. Let $H=N_{G}(E)$. Since $D$ is cyclic, $N_{G}(D) \leq H$. Let $c$ be the block of $k H$ with defect group $P$ such that $c$ is in Brauer correspondence with $b$. By Erdmann's Theorem combined with the compatibility of the Green correspondence and the Brauer correspondence again, the Green correspondence, with respect to vertex $D$, restricts to a bijective correspondence between the isomorphism classes of weight $k H c$-modules and the isomorphism classes of weight $k G b$-modules. Green [8, Theorem 1(ii)] says that the isomorphism classes of simple $k H c$-modules $V$ are in a bijective correspondence with the isomorphism classes of simple $k G b$-modules $U$ whereby $V \leftrightarrow U$ provided $U$ is isomorphic to the socle of the Green correspondent of $V$.

Let $W$ be a weight $k H c$-module, and let $V$ be a simple $k H c$-module. Let $\mathcal{G}(W)$ and $\mathcal{G}(V)$ denote the $k G b$-modules in Green correspondence with $W$ and $V$, respectively. By the previous paragraph, $\mathcal{G}(W)$ is a weight $k G b$-module and $\mathcal{G}(V)$ is an indecomposable $k G b$-module with a unique simple submodule $V_{G}$. Supposing that $W$ and $V$ lie in the same proper component of the $k H$-module $k H / T$ then, by [4, Corollary $5.7(\mathrm{~b})], \mathcal{G}(W)$ and $\mathcal{G}(V)$ lie in the same proper component of the $k G$-module $k G / T \cong{ }_{G} \operatorname{Ind}_{H}(k H / T)$. Plainly, $\mathcal{G}(W)$ and $V_{G}$ lie in the same proper component of $k G / T$. We have shown that, given a weight $k H c$-module and a simple $k H c$-module lying in the same proper component of $k H / T$, then the corresponding weight $k G b$-module and simple $k G b$-module lie in the same proper component of $k G / T$. The required conclusion for $b k G / T$ now follows because, by an inductive argument on $|G|$, we may assume that the required conclusion holds for $c k H / T$. 


\section{References}

[1] J. L. Alperin, Local Representation Theory, (Cambridge Univ. Press, Cambridge, 1986).

[2] J. L. Alperin, Weights for finite groups, Symp. Pure Math. 47(1987) 369-379

[3] L. BARKer, Blocks of endomorphism algebras of modules, PhD Thesis, University of Oxford, Oxford (1991).

[4] L. BARKer, Blocks of endomorphism algebras, J. Alg. 168(1994), 728-740

[5] D. Benson, "Modular Representation Theory: New Trends and Methods", Springer Lecture Notes in Math. 1081, (Springer, Berlin, 1984).

[6] M. Cabanes, Brauer morphism between modular Hecke algebras, J. Alg. 115(1988), 1-31

[7] K. Erdmann, Blocks and simple modules with cyclic vertices, Bull. Lond. Math. Soc. 9(1977), 216-218

[8] J. A. Green, Walking around the Brauer tree, J. Austral. Math. Soc. 17(1974), 197-213

[9] W. Lempken, R. Staszewski, Some 5-modular representation theory for the simple group McL, Comm. Alg. 21(1993), 1611-1629

[10] N. NAEHRIG, Endomorphism rings of permutation modules, J. Alg. 324(2010), 1044-1075

[11] H. SAwADA, A characterization of the modular representations of a finite group with split BN-pairs, Math. Zeit. 155(1977), 29-41

[12] R. Zimmermann, Vertizes einfacher Moduln Symmetrischer Gruppen, PhD Thesis, University of Jena, Jena (2004).

\section{LAURENCE BARKER}

Department of Mathematics,

Bilkent University, 06800 Bilkent, Ankara,

Turkey

e-mail: barker@fen.bilkent.edu.tr

İPEK Tuvay

Department of Mathematics,

Mimar Sinan Fine Arts University,

34380 Bomonti, Şişli, İstanbul,

Turkey

e-mail: ipektuvay@gmail.com

Received: 25 March 2015 